\title{
Ethnologies
}

Chine-Europe-Amérique. Rencontres et échanges de Marco Polo à nos jours. Dirigé par Shenwen Li. (Québec : Presses de l'Université Laval, Collection InterCultures, 2009. Pp. 468, ISBN 978-2-7637-8353-6)

\section{Marie-Hélène Janvier}

Volume 33, numéro 1, 2011

URI : https://id.erudit.org/iderudit/1007807ar

DOI : https://doi.org/10.7202/1007807ar

Aller au sommaire du numéro

Éditeur(s)

Association Canadienne d'Ethnologie et de Folklore

ISSN

1481-5974 (imprimé)

1708-0401 (numérique)

Découvrir la revue

Citer ce compte rendu

Janvier, M.-H. (2011). Compte rendu de [Chine-Europe-Amérique. Rencontres et échanges de Marco Polo à nos jours. Dirigé par Shenwen Li. (Québec : Presses de l'Université Laval, Collection InterCultures, 2009. Pp. 468, ISBN

978-2-7637-8353-6)]. Ethnologies, 33(1), 297-303.

https://doi.org/10.7202/1007807ar d'utilisation que vous pouvez consulter en ligne. 


\section{Comptes RENDUS / Reviews}

Chine-Europe-Amérique. Rencontres et échanges de Marco Polo à nos jours. Dirigé par Shenwen Li. (Québec : Presses de l'Université Laval, Collection InterCultures, 2009. Pp. 468, ISBN 978-2-7637-8353-6)

Cette publication réunit les articles de vingt-deux chercheurs spécialistes des relations entre la Chine et l'Occident. Son maitre d'œuvre, Shenwen $\mathrm{Li}$, a rédigé une introduction générale afin de donner un aperçu de l'histoire de ces relations pour ensuite présenter les trois parties de l'ouvrage : la représentation de la Chine en Occident (13158) ; le christianisme et les activités missionnaires en Chine (161391); et les contacts commerciaux, politiques et culturels (395-468). Cet ouvrage est très bien organisé et structuré puisque le lecteur suit sans difficulté l'articulation entre les parties thématiques et saisit bien la cohérence de l'ensemble. Les études sont fort bien rédigées et documentées; certaines d'entre elles sont complétées par des tableaux, des graphiques et des notes explicatives qui en facilitent la lecture.

La première partie, traitant de l'image de la Chine dans le monde occidental, débute par l'article de Li Zh'an, professeur de l'Université de Nankai en Chine et spécialiste de l'histoire de la dynastie des Yuan, qui s'intéresse au récit tant controversé de Marco Polo. Il se positionne dans le débat toujours d'actualité opposant les spécialistes qui considèrent que ce récit de voyage est une fiction et ceux qui, comme lui, le considèrent comme une réalité historique. Pour ce faire, l'auteur a utilisé des sources chinoises sur les événements historiques de la période mongole afin d'authentifier la véracité des informations trouvées dans le Livre des Merveilles.

Michel Cartier, directeur de l'École des hautes études en sciences sociales de Paris, se penche sur la vision de la Chine qu'entretiennent les Européens entre le XVI et et le XVIII ${ }^{e}$ siècle. Il aborde plus directement la question de l'image de "l'Empire du Milieu » chez les marchands, les explorateurs et les philosophes. S'appuyant sur les écrits de l'époque comme sources de première main, l'auteur constate que les contacts 
initiaux avec cet empire lointain ont eu lieu uniquement dans les ports, donc en périphérie d'une Chine mal connue. Par conséquent, seule une image des marges maritimes et portuaires de la civilisation chinoise parvenait en Europe. Les missionnaires jésuites dépeignirent la Chine tout autrement, car ils purent aller au-delà des ports et pénétrer à l'intérieur de l'empire. L'auteur insiste sur l'importance de ces écrits, qui eurent un impact sur la formation des idéaux philosophiques en France, en nourrissant le débat entre sinophobes et sinophiles ainsi que la recherche d'un nouveau modèle pour la France, voire pour l'Europe toute entière.

Professeure au Département de philosophie de l'Université de Montréal, Anna Ghiglione vise à exposer la perception et la réception de la pensée philosophique chinoise en Occident, du XVII siècle à nos jours. Son étude concise et complète lui permet de déterminer que le contexte historique, qu'il soit chinois ou occidental, joue un rôle clé dans la vision de la pensée philosophique chinoise au-delà de l'Orient. Elle emploie d'ailleurs le terme «inséparable» pour qualifier l'importance du lien entre l'évolution des contacts Orient-Occident et l'adoption de la philosophie chinoise au sein de ce dernier monde.

Paul Servais, professeur de l'Université catholique de Louvain, s'est intéressé à un personnage spécifique, Charles de Harlez de Deulin, fondateur des études sinologiques dans cette même université. L'auteur s'interroge sur les représentations élogieuses et flatteuses de la Chine transmises par ce dernier, plus particulièrement dans sa présentation de la poésie chinoise. Or, toujours selon Paul Servais, Harlez avait une connaissance incomplète de ce pays lointain puisqu'il n'avait eu entre les mains qu'une infime partie des textes disponibles, qui étaient pour la plupart retravaillés ou en partie censurés. Au fil de ses écrits, ce dernier aurait tenté de faire renaître la sinophilie au sein d'une époque clairement sinophobe, le XIX ${ }^{\mathrm{e}}$ siècle.

L'étude d'Angel Pino, professeur à l'Université Michel de Montaigne à Bordeaux ainsi que directeur du Centre d'études et de recherches sur l'Extrême-Orient de ce même établissement, porte sur la réception des œuvres de $\mathrm{Pa}$ Kin en France. Il se consacre spécifiquement à leurs rééditions et traductions en langue française depuis la parution de la première d'entre elles, en 1921. Son étude recense l'évolution des différentes éditions francophones des romans de Pa Kin. Angel Pino cherche à démontrer dans son article que cet auteur a, au fil des années, 
acquis une certaine notoriété dans le monde de la publication. Constituée de simples notices biographiques ou d'études générales entre 1978 et 1990, son œuvre est devenue l'objet d'études plus approfondies à la fin du XXe siècle.

David van den Abbel, de l'Université catholique de Louvain, examine la perception de la Révolution culturelle chinoise en Belgique francophone par l'entremise de six quotidiens belges. L'idée générale de l'auteur est de déterminer la manière dont la presse faisait écho à la situation en Chine afin de saisir l'opinion de la population belge envers ce pays. Il considère que les quotidiens sont le miroir de la pensée populaire. Il constate que la vision de chacun d'eux à propos de la Révolution culturelle n'a pas été teintée de leur idéologie politique respective puisque la compréhension de cet événement et l'image qu'ils en ont transmise furent similaires, étant largement construites sur la peur qu'éprouvait l'Occident devant le danger communiste que représentait alors Mao Zedong.

Serge Granger, professeur à l'Université de Sherbrooke, traite de l'œuvre cinématographique québécoise et du rôle clé qu'a joué la Chine dans son évolution. Le but de l'auteur est de mettre en lumière le travail accompli par les missionnaires dans la naissance du cinéma québécois, à une époque où plusieurs centaines d'entre eux sont envoyés en Orient pour faire connaître le peuple chinois à la population québécoise. Serge Granger traite aussi de l'image de la Chine dans le septième art au Québec et de son évolution entre 1930 et 1980, c'est-à-dire avant la réouverture de la Chine à l'Occident.

La deuxième partie, consacrée aux interactions entre christianisme et confucianisme par l'entremise des activités missionnaires, est la plus volumineuse de l'ouvrage. Jean Charbonnier, prêtre des Missions étrangères de Paris et docteur ès lettres en études chinoises, donne le ton à cette partie en traitant de l'influence de la Chine sur le christianisme. L'auteur aborde la progression de la sinisation de la religion chrétienne depuis sa toute première implantation jusqu'à nos jours. Selon Jean Charbonnier, christianiser la Chine ou siniser le christianisme représentent deux ambitions utopiques dans la mesure où, d'une part, convertir tous les Chinois est un dessein irréaliste et que, d'autre part, l'Occident ne semble pas prêt à accepter le confucianisme au sein de sa religion prédominante. 
Jean-Paul Wiest, membre de l'Institut Ricci à San Francisco et chercheur du Beijing Center for Chinese Studies, propose une réflexion sur l'état de l'implantation chrétienne en Chine depuis l'ouverture progressive de celle-ci à la fin des années 1970. Il tend à démontrer que, malgré les échecs et les déboires du passé, les contacts entre la Chine et l'Occident ont évolué pour permettre une patiente intégration du christianisme à la culture chinoise. De fait, après le renouvellement du Front uni et devant la nécessité de la modernisation, une intégration plus « lumineuse » du christianisme s'avérait indispensable à la «nouvelle société socialiste chinoise ».

Professeure à l'Université de la Rochelle, Martine Raibaud étudie la fondation d'écoles catholiques sur le territoire chinois depuis la première Guerre de l'Opium jusqu'à l'aube du XX $X^{\mathrm{e}}$ siècle. Elle s'est attardée plus spécialement aux personnages qui ont joué un rôle déterminant dans l'implantation des collèges catholiques et dans le courant de modernisation, comme les frères $\mathrm{Ma}$, Laurent $\mathrm{Li}$, les pères maristes et Ying Lianzhi.

Le professeur Paul Rule, membre du Macau Ricci Institute et professeur à l'University of San Francisco, ainsi que Zhang Xiping, directeur du Centre d'études sur la sinologie d'outre-mer de l'Université des études internationales de Beijing (Beiwai), ont tous deux travaillé sur les échanges entre l'empereur Kangxi et les missionnaires jésuites présents en Chine. Dans son article, Rule se lance dans un débat fort complexe sur la question d'une occasion manquée entre le Fils du Ciel et les missionnaires. En fait, l'auteur s'affiche en défenseur de Kangxi, écrivant qu'il était œcuméniste avant l'heure et qu'il était prêt à inclure les idées chrétiennes dans son empire. Or, s'il était tolérant envers ces dernières, c'était pour nourrir sa curiosité pour les connaissances européennes. Rule écrit que l'un des responsables de ce «rendez-vous manqué » était Rome, et sa bulle papale transmise par Maillard de Tournon qui discréditait les traductions ou adaptations chinoises du concept de Dieu, ou de Dieu unique. Quant à l'étude de Xiping, elle s'intéresse à l'implication de l'empereur Kangxi dans le travail de Joachim Bouvet, soit son étude du Livre des mutations. De par cette intervention, l'auteur s'attache à démontrer que Kangxi manifestait un intérêt considérable pour les connaissances occidentales et que, par le fait même, ce dernier souhaitait que Bouvet saisisse la culture chinoise dans toute sa splendeur. 
Sun Juo Yi, directeur de l'Universal Education Center à l'Université de Yuan Pei à Taiwan, aborde les influences occidentales sur l'architecture chinoise. Il s'arrête sur les mariages de l'art chinois et occidental dans les pavillons composant le Palais d'été Yuanmingyuan, soit celui construit sous la dynastie de Qianlong. L'auteur fait ressortir l'implication des Jésuites dans cette transmission d'idées architecturales en provenance de l'Occident.

Professeur de l'Université d'Ottawa, Jean-Guy Daigle fait porter son enquête sur le centre de la Chine, dans la province du Sichuan oriental, afin d'étudier les manifestations antichrétiennes qui s'y sont produites dans la seconde moitié du XIX e siècle. Selon l'auteur, le peu de conversions était dû à trois principaux facteurs : la barrière de la langue chez la plupart des missionnaires, le manque d'intérêt pour la religion chrétienne chez les Chinois et le handicap que créait l'association forcée des hommes de foi avec l'impérialisme des grandes puissances européennes.

Spécialiste de l'histoire de la Chine moderne au Collège de France, Pierre-Étienne Will affine l'analyse des relations Orient-Occident en étudiant les démêlés entre le magistrat Ma Yugui, les missionnaires jésuites du Zhili et, également, les ennemis de ces derniers, les Boxers ou «poings fermés ", suite à la prise de Pékin par les corps expéditionnaires de l'Alliance des huit nations. Pour ce faire, l'auteur a utilisé la correspondance officielle et les proclamations adressées à la population ou au personnel subalterne. Il constate que les documents de 1900 et de 1901 contiennent un nombre important de textes traitant de la relation entre les Jésuites et les Boxers.

Deux articles sont consacrés aux activités des missionnaires québécois en Chine. Shenwen Li, professeur au département d'histoire de l'Université Laval, se penche quant à lui sur un pan de l'histoire très peu étudié, la mission en Chine des Jésuites québécois entre 1918 et 1945. Il survole habilement la mission chrétienne dans la région de Xuzhou en décrivant les difficultés auxquelles les Jésuites ont eu à faire face dès l'arrivée des premiers d'entre eux jusqu'à la capitulation japonaise au milieu des années 1940. Avec cet article, l'auteur souhaite attirer l'attention sur l'importance des missionnaires canadiens-français en terre chinoise. De son côté, Diana Lary, professeure au département d'histoire à l'Université de la Colombie-Britannique, a étudié spécifiquement l'aide apportée par les missionnaires québécois durant 
les horreurs de l'occupation japonaise. Elle a souligné le courage de ces hommes de foi devant la dureté de cette occupation.

John David Meehan, chercheur jésuite au Campion College, aborde le cas particulier du Jésuite français Robert Jacquinot de Bésange qui avait instauré une zone de protection pour les réfugiés à Shanghai durant la guerre sino-japonaise. L'auteur traite habilement de cet effort humanitaire qui a permis de protéger de nombreuses victimes de guerre et qui a inspiré les missions ultérieures. Avec cet article, il souhaite faire connaître cette "zone Jacquinot » qui semble avoir été oubliée par les historiens.

L'ouvrage se termine par une troisième partie relativement plus brève portant sur les contacts commerciaux, politiques et culturels entre la Chine et l'Occident. Dans son étude, Kathy Boulet, diplômée en histoire à l'Université Laval de Québec, montre comment le botaniste Robert Fortune a découvert les secrets du thé chinois, contribuant ainsi à briser le monopole commercial de la Chine. Carl Dery, doctorant de l'Université Laval, traite des facteurs politiques du déclenchement de la première Guerre de l'Opium en s'arrêtant sur les causes du conflit et sur ses ramifications réelles. L'auteur propose une nouvelle perspective sur les enjeux justifiant l'entrée en guerre de la Grande-Bretagne contre la Chine en mettant l'accent sur les facteurs politiques et diplomatiques du déclenchement des hostilités plutôt que sur les aspects économiques, à rebours de la perspective qui prévalait jusqu'ici.

L'historien et journaliste Chen Yan traite de la réintroduction de l'Occident en Chine dans les années 1980 après plus de deux décennies d'emprise maoïste. Ce dernier explique que la population chinoise, plus spécifiquement la jeunesse, est avide de nouvelles connaissances, car elle souffre de la «famine intellectuelle et spirituelle » que lui a léguée la Révolution culturelle. Ce virage culturel serait dû, selon l'auteur, à un besoin vital de la société.

L'article de Sun Min, doctorante de l'Université Laval, conclut ce volet en traitant du rôle des traducteurs dans la diffusion de la littérature québécoise en Chine depuis 1980. L'auteure s'attarde aux procédés de traduction, aux genres littéraires traduits et à leur succès en Chine.

Cet ensemble de textes riches et variés permet de mettre en lumière les différentes facettes des relations diversifiées et complexes entre la Chine et l'Occident depuis l'époque de Marco Polo jusqu'à nos jours. 
Fruit d'une belle collaboration entre des chercheurs occidentaux et ceux du monde chinois, dont certains grands spécialistes de la Chine, cette nouvelle publication représente en elle-même le résultat de fructueux échanges scientifiques sino-occidentaux et une contribution importante à l'avancement des recherches en histoire des relations entre la Chine et l'Occident.

Marie-Hélène Janvier

Université Laval

Éthlque des rapports Nord-Sud. Regards crolsés. Dirigé par † Gérard Verna et Florence Piron (Québec, 2010, Presses de l'Université Laval. Pp. 380. ISBN : 978-2-7637-8992-7).

L'ouvrage Éthique des rapports Nord-Sud. Regards croisés est paru en 2010 aux Presses de l'Université Laval. Si le titre de l'ouvrage est déjà en lui-même très évocateur, l'image de la page de couverture est aussi significative puisqu'elle représente une foule en liesse portant des couleurs différentes et allant dans une même direction. Elle évoque l'idée que le monde est constitué de pays de tailles et de poids politiques et économiques différents, mais que tous sont appelés à collaborer de manière équitable, puisqu'ils ont tous des destins communs.

Paru sous la direction du regretté Gérard Verna, professeur titulaire au Département de management de l'Université Laval, et de Florence Piron, professeure agrégée au Département d'information et de communication, avec la collaboration de Corinne Béguerie, cet ouvrage de 362 pages se veut un manuel de réflexion sur la composante éthique des relations entre le Nord et le Sud, ou entre les riches et les moins riches. Divisé en quatre parties, cet ouvrage est constitué de seize contributions toutes plus originales les unes que les autres. Ses différents auteurs se sont intéressés à ce qu'est l'éthique et ses enjeux en économie et en politique. En passant en revue les grandes crises économiques du monde actuel, les dérives boursières, les scandales politiques, les délits d'initiés, la corruption, ainsi que diverses situations pratiques à travers le monde, ces auteurs n'ont pas hésité à pointer du doigt les inégalités entre les pays du Nord et ceux du Sud. Dans leurs propos, il est 